\title{
Unraveling atomic motions through Dynamic Pair Distribution Function Analysis
}

\author{
Allyson Fry-Petit, California State University Fullerton
}

Direct access to the atomic motions of vibrational excitations has long been of interest due to the connection between phonons and physical properties. Indirect methods, such as vibrational spectroscopy and multidimensional methods, are routinely used to assign molecular motions in molecules and solid-state materials, however dynamic pair distribution function (DPDF) analysis via inelastic neutron scattering finally provides direct real space resolution to the local atomic motions of phonons. In this work we outline the advances in the data treatment of inelastic neutron data for the use in DPDF. We will outline the development of normal mode analysis coupled to least-squared regression being used in our lab to elucidated the local atomic vibrations parsed out from DPDF. We have applied DPDF to several materials of interest spanning a wide variety of properties potentially effected by local vibrations, including orbital ordering that quenches a paramagnetic moment $\left(\mathrm{La}_{4} \mathrm{Ru}_{2} \mathrm{O}_{10}\right)$, superconductivity $(\mathrm{FeSe})$, and luminescence $\left(\mathrm{Y}_{3} \mathrm{Al}_{5} \mathrm{O}_{12}: \mathrm{Ce}\right)$, will be presented. 